\title{
How to correct and prevent mid-face widening after using floating (or non-fixation) type polydioxanone thread-lift
}

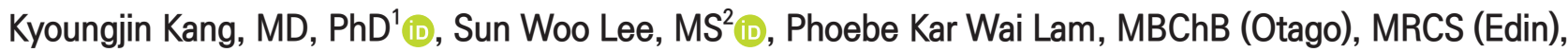 \\ MScPDerm (Cardiff) ${ }^{3}$ i⿺
}

${ }^{1}$ Seoul Cosmetic Surgery, Busan, Rep. of Korea, ${ }^{2}$ Bison Medical, Seoul, Rep. of Korea, ${ }^{3}$ Perfect Skin Solution, Hong Kong

\begin{abstract}
Thread-lifting is a minimally invasive face-lifting procedure that lifts and reposition ptotic soft tissue associated with ageing. This procedure has become increasingly more popular in the last decade. mid-face widening (MFW) is a frequent complaint after threadlifting, as the descended ptotic soft tissues gathered to the middle of the thread during repositioning. The bunching up of the lax soft tissue increases both the facial volume and the MFW. We attempt to overcome the MFW as an unexpected common complication. Three patients underwent our thread-lifting procedure and followed up for six months. The assessment parameter, bizygomatic diameter (BZD), taken at baseline, three and six-month follow-up with standardised digital computerised 3-dimensional (3D) images using MIRAGE 3D, Taiwan. The floating $90 \mathrm{~mm}$ and $115 \mathrm{~mm}$ threads, were inserted in the following sequence: first, two single-layers (temporal and high temporal, along the superior temporal line) and then, one dual-layer (zygomatic/cheek region). This thread arrangement aims to overcome and correct the position-dependent downward sagging and volume loss associated with facial ageing, but without increasing the MFW of the patients. None of the patients developed MFW following thread-lifting at three- and six months following treatment. An observed reduction between 4.7-17.9 mm (average, $11.3 \mathrm{~mm}$ ) in BZD recorded at six-month followup. There was no significant discomfort or complications have been reported. Based on the results of our cases, this floating technique, combining two single and one dual-layer, in the three regions (temporal, high temporal and zygomatic/cheek) appears favourable in achieving satisfactory face-lifting and rebalancing facial volume without an increase in MFW.
\end{abstract}

Keywords: aging; polydioxanone; rhytidoplasty; sutures; zygoma

\section{Introduction}

Facial recontouring via the thread-lift procedure counteracts the sagging soft tissue with ageing. Firstly, by mechanically lifting following threads insertion, and if ptotic soft tissue repositioned correctly, a reversal of the course of descent of ptotic soft tissue, a rebalancing of facial volume as well as facial recontouring, will occur.
Secondly, a few weeks after insertion, the implanted threads will stimulate new collagen formation, which continues to sustain the lifting results. Thirdly, the deposited sutures trigger a cascade of cellular reactions, known as mechanotransduction, that intensify collagen production, fibrous tissue adhesion, increased elasticity, tissue tightening that resulting in enhanced facial lifting and skin regeneration [1].

Thread-lifting techniques are generally differentiated by

Received October 8, 2020; Revised November 22, 2020; Accepted November 26, 2020

Corresponding author: Phoebe Kar Wai Lam

E-mail:drlamkarwai@gmail.com

This is an Open Access article distributed under the terms of the Creative Commons Attribution Non-Commercial License (http://creativecommons.org/licenses/by-nc/4.0), which permits unrestricted non-commercial use, distribution, and reproduction in any medium, provided the original work is properly cited.

Copyright @ 2020 Korean Society of Korean Cosmetic Surgery and Medicine (KSKCS \& KCCS). 
whether the proximal end of the thread is "anchored (fixed) or "floating (non-fixed) in the tissue. For our cases, the inserted non-fixed or floating suture-threads latch onto the zygomatic ligament (true ligament) and the temporal septal fascia and gather the ptotic soft tissue into the middle part of the threads resulting in reposition with facial recontouring [2]. However, improper thread placement can create an "iatrogenically unbalanced face that exaggerates pre-existing deformity (e.g., temporal hollowing).

Our unique floating thread-lift technique aims explicitly to reverse the descent of ageing soft tissue that leads to better facial contouring without mid-face widening (MFW) and prevent secondary upper facial sagging by the addition of vertical temporal reinforcement.

\section{Case report}

Three patients between (30-65 years old) with good skin elasticity, an adequate layer of soft tissue, and no history of keloid and no recent surgery or thread-lifting for face in the past six months. All thread-lift procedures were performed with the patients under local anesthesia in an outpatient operating room.

\section{Design (thread insertion)}

Polydioxanone (PDO) bidirectional cog threads (Stellar 1; Cosmedic, a subsidiary of Bison Medical Ltd., Seoul, Korea), 90 and $115 \mathrm{~mm}$ in length, were used in this study, along with a 60$\mathrm{mm} 23 \mathrm{G}$ needle. A total of 16 suture-threads inserted into each case, eight sutures on each side of the face in the following arrangement (Fig. 1).

Single plane: a pair of $90 \mathrm{~mm}$ threads are inserted superficially, within the high temporal area, along the superior temporal line (STL), and the zygomatic/cheek region.

Dual plane: two $90 \mathrm{~mm}$ threads inserted superficially and two $115 \mathrm{~mm}$ inserted in the deep plane, within the temporal region.

\section{Preparation}

The frontal branch of the facial nerve travels within the temporoparietal fascia (TPF) but is more superficial when it crosses the proximal part of the zygomatic arch into the temporal region. The temporal branch of the facial nerve runs deep beneath TPF and the superficial musculoaponeurotic system (sub-SMAS) and runs along the zygomatic arch. The superficial temporal artery, from superior border of parotid gland, travels superficially across the distal part of the zygomatic process into the temporal region. A thorough understanding of the underlying anatomy when performing a traumatic procedure with a blunt cannula may reduce the risk of inadvertent injuries to vessels and nerves during thread insertion.

\section{Anesthesia and entry points}

Following draping using a standard sterile technique, all entry points were infiltrated with $0.1-2 \mathrm{ml}$ of $1 \%$ lidocaine with adrenaline. An 18G needle was used to create stab incisions at the three entry points on each side of the face: the suprazygomatic, temporal, and high temporal areas (Fig. 1).

\section{Thread-lift procedure}

Step 1: Zygomatic/cheek area (single layer)

The 90-mm threads were inserted into the skin through the

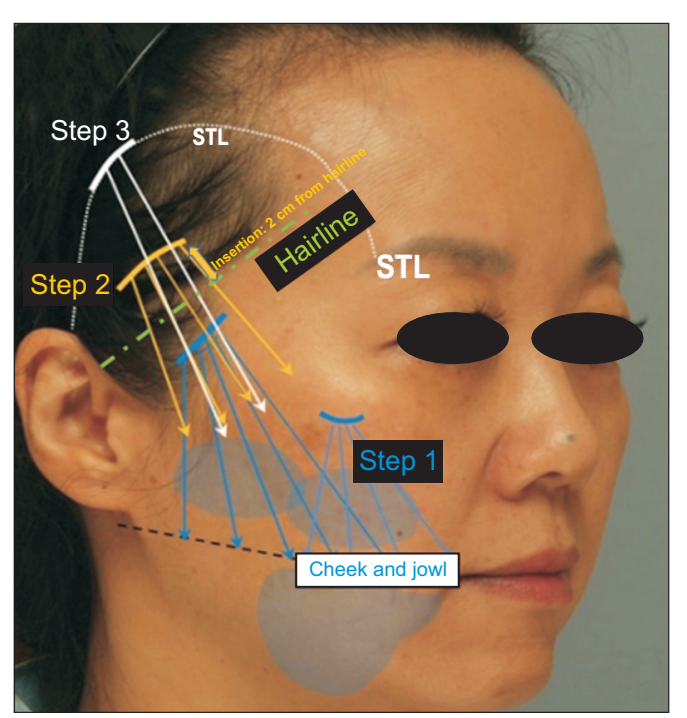

Fig. 1. This diagram shows the entry points and vector direction to which the threads are placed for different steps. Step 1 (zygomatic/ cheek to jowl): The $90 \mathrm{~mm}$ threads are inserted into the skin, above the zygomatic arch, passing through the superficial fat pad system and ligament (zygomatic retaining ligament, false ligament and connective tissue bands [septa]). Step 2 (temporal): The $90 \mathrm{~mm}$ and $115 \mathrm{~mm}$ threads are inserted into the skin (entry point) in the temporal area, about $10 \mathrm{~mm}$ from top of helix, $2 \mathrm{~cm}$ above and parallel to the hairline. Threads inserted in a dual-plane manner, (superficial and deep to superficial musculoaponeurotic system [SMAS]), above the zygomatic arch, passing through ligaments (inferior temporal, zygomatic cutaneous and masseteric cutaneous ligament) and superficial fat in the cheek and jowl. Step 3 (high temporal, STL): The $90 \mathrm{~mm}$ threads are inserted into the skin along the superficial temporal line (entry point) into in a deep plane between the superficial temporoparietal fascia and the deep temporal fascia, which fuse and merge into the plane at the level between the superficial fat (subcutaneous) and the superficial muscular aponeurotic system. STL: superior temporal line. 
entry point above the zygomatic arch. The suture threads were then passed through both the superficial fat pad system (medial cheek, nasolabial area, and superficial jowl) and the ligaments (zygomatic retaining ligament and false ligament) as well as the connective tissue bands (septa) in sequence (Fig. 1, 2). The ligament and connective tissue provide anchoring points for a robust mechanical lift (Fig. 1, 2).

\section{Step 2: Temporal (dual plane)}

The 90- and 115-mm threads were inserted into the temporal area, approximately $10 \mathrm{~mm}$ from the top of the helix and $2 \mathrm{~cm}$ above and parallel to the hairline (entry point). The threads introduced into the entry point above the zygomatic arch in two planes (superficial and deep to the SMAS), passing through ligaments (inferior temporal, zygomatic cutaneous, and masseteric cutaneous) and superficial fat in the cheek and jowl. Step 2 strengthens the mechanical lifting obtained from step 1.

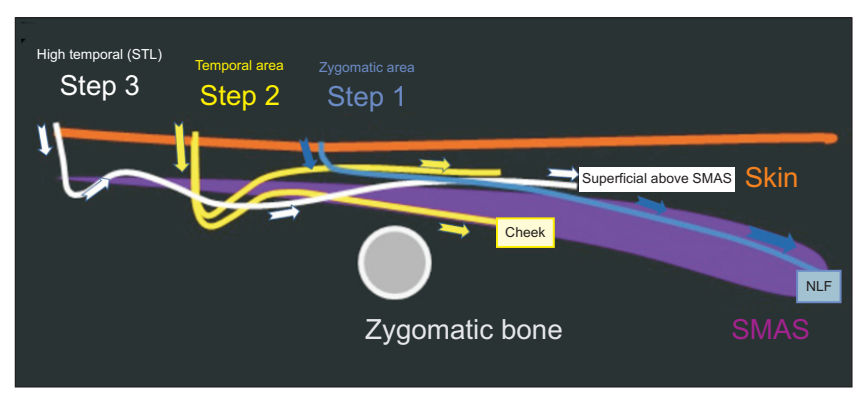

Fig. 2. Diagramatic representation of the design of thread-layering in their respective placement plane. STL, superior temporal line; SMAS, superficial musculoaponeurotic system; NLF, nasolabial fold.

\section{Step 3: high temporal (superior temporal line)}

The 90 -mm threads were inserted into the skin at any point along the STL (entry point) and into the deep plane between the superficial temporoparietal fascia (STF) and the deep temporal fascia (DTF). The two fasciae merge into the plane between the superficial fat (subcutaneous) and the SMAS. The DTF provides anchor points for the strong mechanical lift, reinforcing the lift from steps 1 and 2, and fixation for the middle and lower face (Fig. 1, 2).

\section{Results}

None of the three patients underwent the thread-lift procedure, developed MFW at 3 and 6 months after the procedure. A reduction between 4.7 and $17.9 \mathrm{~mm}$ (average, $11.3 \mathrm{~mm}$ ) of the bizygomatic diameter (BZD) was observed at the 6-month follow-up. Transient discomfort, which includes immediately after thread insertion, as well as palpable thread end were reported. Both resolved conservatively. No significant pain or complications (e.g., dimpling or infection) were reported (Fig. 3-5).

\section{Discussion}

The characteristic signs of facial aging are the result of several contributing factors, including the descent of soft tissue, facial fat volume deflation and ligamentous laxity. Bony resorption further weakens midface support causing loss of overall facial height. MFW with transient swelling is possible following the thread-lift procedure by which descended soft tissue is repositioned and anchored along the zygomatic arch.
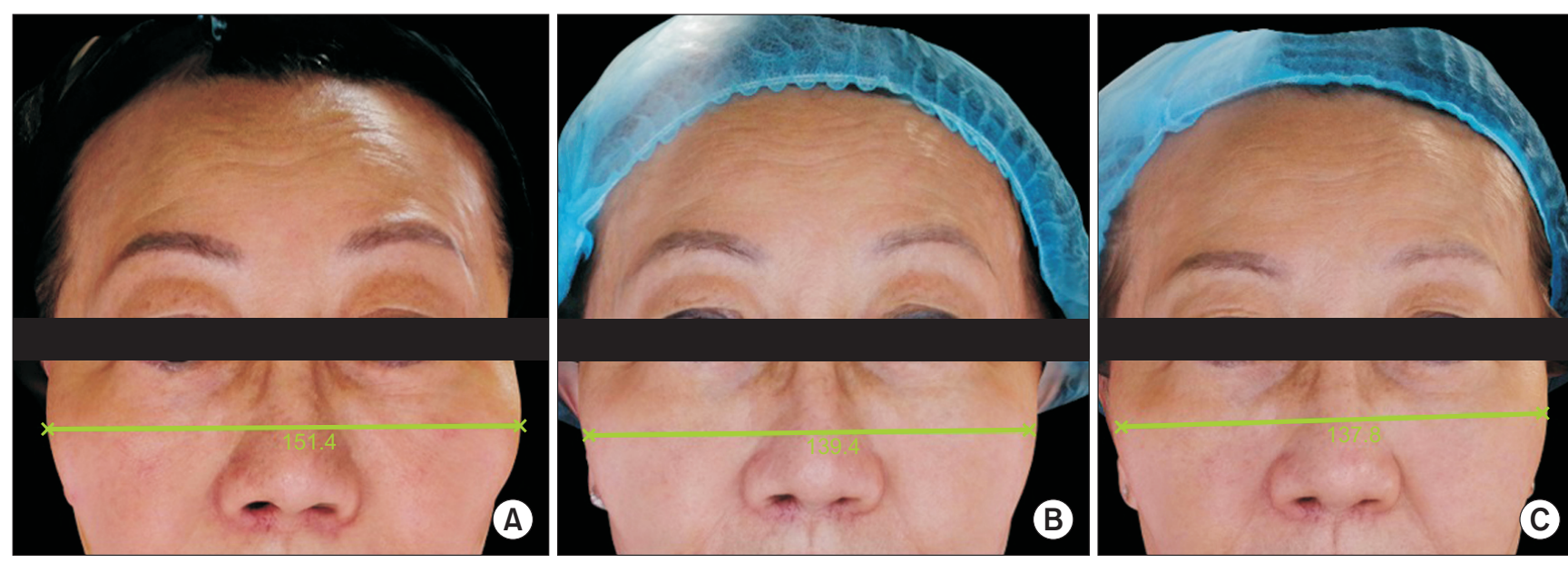

Fig. 3. A 62-year-old female patient underwent thread-lift with our technique, for improving facial contouring. This patient has mild-tomoderate soft tissue laxity, bizygomatic diameter (BZD) of $151.4 \mathrm{~mm}$. The above pictures were taken before treatment (A), at 3-months (B) and 6-months (C) following thread-lift. Her overall facial contour has improved, and skin appears firmer with the lifting. The measured reduction of BZD was $12 \mathrm{~mm}(\mathrm{~B})$ and $13.6 \mathrm{~mm}(\mathrm{C})$ when compared to baseline measurements. 

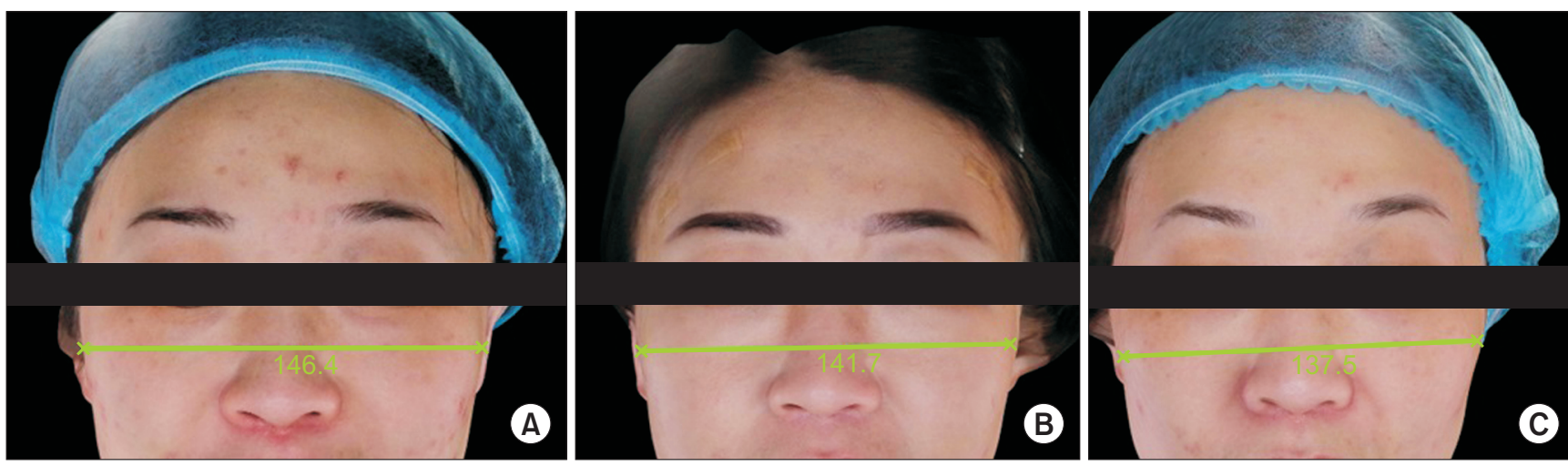

Fig. 4. A 52-year-old female patient, body mass index of $25 \mathrm{~kg} / \mathrm{m}^{2}$, moderate mid-to-lower face laxity, and prominent temporal hollowing who underwent thread-lifting using this technique and botulinum toxin (60 IU, Dysport, UK) to masseter muscle (both side). The above pictures were taken before treatment (A), at three-months (B) and six-months (C) following thread-lift. There was considerable improvement in inner overall facial contour (mid-to-lower face and jawline) was more well-defined and lessening of the nasolabial fold. The measured reduction of bizygomatic distances were $4.7 \mathrm{~mm}$ (B) and $8.9 \mathrm{~mm}$ (C) when compared to baseline measurements. There was no significant discomfort even though one of the thread ends was temporarily palpable (because of thin skin) which resolve conservatively around 1 month following the procedure.
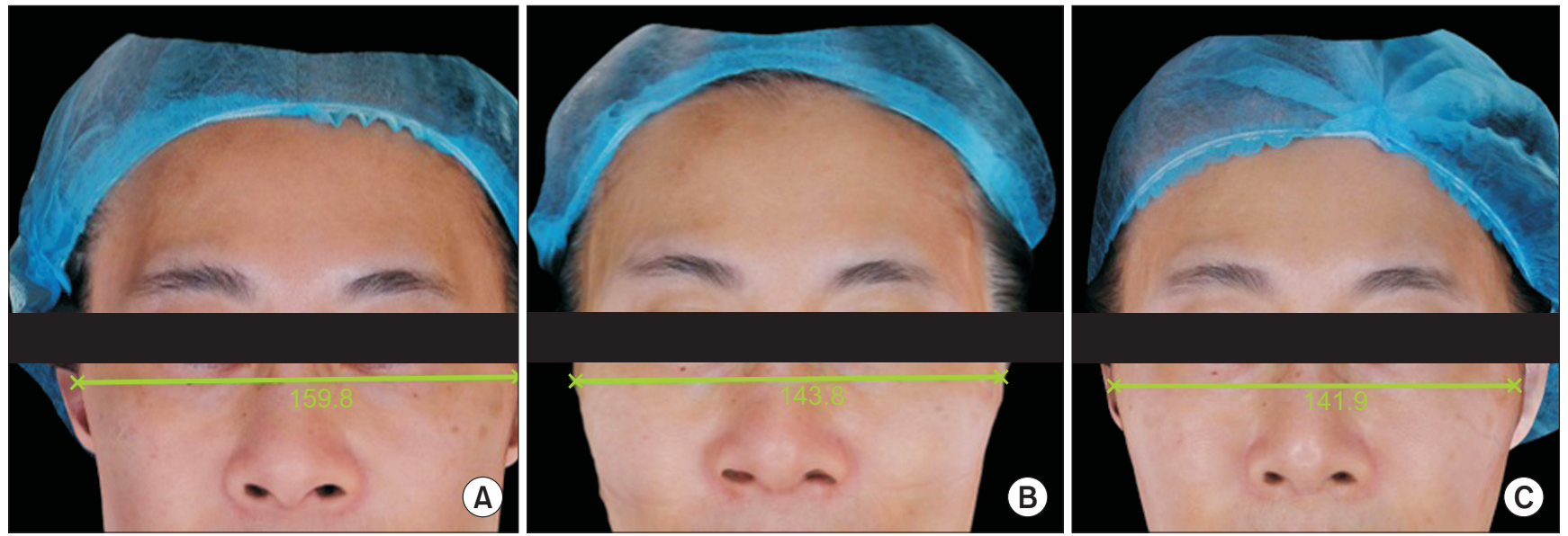

Fig. 5. A 42-year-old female patient with moderate skin laxity who underwent thread-lifting with this technique. The above pictures were taken before treatment (A), at 3-month and 6-month following thread-lift. Lifting was notable at 3 months but more pronounced by 6 months, and the measured reduction of bizygomatic distances was $16 \mathrm{~mm}$ (B) and $17.9 \mathrm{~mm}$ (C) when compared to baseline measurements. She did not experience any significant discomfort apart from some tightness within the upper face and temporal that resolved within one week after treatment.

Some face-lift techniques [3-5] emphasized the re-creation of facial fullness by layering the SMAS. Others reported regardless of facial lifting techniques, there is no net increase in facial volume without importing additional tissue from donor site [6,7]. The design of restorative vectors are also crucial in preventing the development of MFW and iatrogenic facial imbalance when repositioning ptotic soft tissue in face-lifting procedures, particularly in short round faces [3].

This combined thread-suture placement design in both single-plane (zygomatic and high temporal region) and dual-plane (temporal region) thread-suture placement aim to lift descend- ed tissue in the superior-posterior direction in three vector dimensions. Therefore, better reversing the course of soft tissue descent with ageing, together with the vertical repositioning via the high temporal lifting, this configuration prevents secondary upper-face sagging that gives rise to MFW after the thread lift. Although MFW following a face-lift or thread-lift procedure has not been widely reported, it is one of the most frequent complaints. Hence, the risk of MFW should be discussed with the patient before the procedure to manage expectations.

Another point to consider for the overall success of thread sutures is the balance between the weight of the sagging tissue 
(overall loading) and the total number of thread sutures applied. The overall strength of the lift is proportional to the number of sutures used. The overall load of descended tissue is distributed evenly over the sutures with less strain on any one suture lifting point and, more importantly, more durable results.

Our case series included three consecutive patients with mild-to-moderate facial laxity and no history of having undergone a face-lift procedure 6 months before treatment. We aimed to determine if MFW (defined as an increase in the BZD) occurred 3 and 6 months after our thread-lift procedure. All patients did not report any significant discomfort or complications. Clinical observation and measurements obtained via 3-dimensional computerized digital imaging before and after treatment showed no evidence of MFW in any patient.

The major limitation of a case series is the lack of controls, and thus can be prone to bias. Nevertheless, based on the findings of this case series, a more extensive study with a longer follow-up period should be conducted to determine whether the changes are statistically significant.

\section{Conclusion}

The success of the thread-lift procedure depends on the biomechanics of the threads that underlie structural supports, complex tissue interaction, and the ability of the patient to synthesize new collagen.

This floating thread-lift technique lifts the SMAS in two planes (superficial and deep) is effective in reversing the natural course of soft tissue descent, rebalancing volumetric changes. This arrangement is further combined with a high temporal vertical lift, which serves to prevent the development of sec- ondary upper-face sagging following the thread lift. The overall final result is a more natural, evident and longer-lasting lift, and without the development of MFW.

\section{Conflicts of interest}

All the PDO material suture threads (Stellar 1) were supplied and sponsored by Cosmedic, Bison Medical Ltd., Seoul, Korea.

\section{References}

1. Hwang U, Lee J, Kang K. Quantitative analysis of polydioxanone thread effects on lid-cheek junction. J Cosmet Med 2018;2:22-6.

2. Kim B, Oh S, Jung W. The art and science of thread lifting: based on pinch anatomy. Singapore: Springer; 2019. p. 9-10.

3. Stuzin JM, Baker TJ, Baker TM. Refinements in face lifting: enhanced facial contour using vicryl mesh incorporated into SMAS fixation. Plast Reconstr Surg 2000;105:290-301.

4. Stuzin JM. Restoring facial shape in face lifting: the role of skeletal support in facial analysis and midface soft-tissue repositioning. Plast Reconstr Surg 2007;119:362-76.

5. Rohrich RJ, Ghavami A, Lemmon JA, Brown SA. The individualized component face lift: developing a systematic approach to facial rejuvenation. Plast Reconstr Surg 2009;123:1050-63.

6. Guerrerosantos J. Simultaneous rhytidoplasty and lipoinjection: a comprehensive aesthetic surgical strategy. Plast Reconstr Surg 1998;102:191-9.

7. Swanson E. Malar augmentation assessed by magnetic resonance imaging in patients after face lift and fat injection. Plast Reconstr Surg 2011;127:2057-65. 